\title{
Quantum Percolation of One-Electron States in Power-Law Diluted Chains
}

\author{
M. P. da Silva Jr., S. S. Albuquerque, F. A. B. F. de Moura, and M. L. Lyra \\ Instituto de Física, Universidade Federal de Alagoas, Maceió AL 57072-970, Brazil
}

Received 22 October, 2007

\begin{abstract}
We investigate the nature of one-electron eigenstates in power-law diluted chains for which the probability of occurrence of a bond between sites separated by a distance $r$ decays as $p(r)=1 / r^{1+\sigma}$. Using an exact diagonalization scheme on finite chains, we compute the spreading of an initially localized wave-packet, the time dependent participation number as well as the return probability. Our results show the existence of a phase of extended states. By considering the scale invariance of the fluctuations of the participation number at the Anderson transition, we obtained that extended states emerges for $\sigma<0.68$. This limiting value is larger than the one reported in the literature for the emergence of extended states in one-dimensional Anderson models with power-law decaying couplings.
\end{abstract}

Keywords: Quantum percolation; Anderson localization; Long-range couplings

I. INTRODUCTION

The Anderson localization theory describes some relevant aspects concerning the properties of non-interacting electron systems with uniformly distributed disorder. In onedimensional $(1 D)$ and two-dimensional $(2 D)$ electronic systems with short-range hopping, the scaling theory $[1,2]$ predicts the absence of a disorder-driven metal-insulator transition (MIT) for any degree of uncorrelated disorder. On the other hand, when long-range couplings are assumed, a transition from localized to delocalized electronic states can be found even in 1D disordered systems [3, 4]. In this case, one has an interplay between the hopping range and the degree of disorder. The former favors propagation while the later inhibits it. It is worthwhile to mention that propagation of carriers was also obtained in low-dimensional models with short-range hopping but presenting correlated disorder, such as random dimer chains [5-8] and in chains with scale-free disorder[9-11], as well as in chains containing quasi-periodic structures, as for example Fibonacci, Thue-Morse and Harper sequences [12-14].

It was shown that an ordered $1 \mathrm{D}$ system with hopping terms decaying with a power-law characterized by an exponent $\alpha$ recovers the result for the tight-binding model for $\alpha>2$ [15]. More interesting is the behavior corresponding to $0<\alpha<1$. For $\alpha=0$ an initially localized wave-packet presents self trapping, i.e., the particle performs oscillations in a definite region of the lattice, visiting periodically the starting position. By increasing $\alpha$, the localization is lost. When the power exponent equals unity, and for sufficient short times, the wave-packet diffuses with a diffusion coefficient that increases with the number of sites. This effect is absent in the model with only nearest-neighbor hoppings [15].

More recently, the dynamics of an electron in the onedimensional Anderson model with non-random hoppings falling off as some power $\alpha$ of the distance between sites was investigated [16]. It was found that the larger the hopping range, the more extended the wave-packet becomes as time evolves. When the disorder is increased, the wave-packet tends to remain more localized. For a low degree of disorder, the exponent $\alpha=1.5$ indicates the onset for fast propagation.
Moreover, the inclusion of a dc electric field introduces the effect of dynamical localization. The fast propagation found for $\alpha<1.5$ is in agreement with the reported delocalization of states located close to one of the band edges [17-19].

The power-law random band matrix (PRBM) model also exhibits a delocalization transition [3, 4]. This model describes one-dimensional electronic systems with random long-range hopping amplitudes with standard deviation decaying as $1 / r^{\alpha}$ for sites at a distance $r>>b$, where $b$ is a typical bandwidth. It was shown that at $\alpha=1$, this model presents an Anderson-like transition with all states being localized for $\alpha>1$ and extended for $\alpha<1$. At the critical point $\alpha=1$, the inverse participation ratio distribution, the wavefunctions multifractal spectra, the level statistics and the timeevolution of the wave-packet size have been investigated both analytically and numerically [3, 4, 20-23]. Within the same spirit of the PRBM, a model for non-interacting electrons in a $2 D$ lattice with random on-site potentials and random powerlaw decaying transfer terms was numerically investigated by exploring the finite-size scaling properties of the fluctuations in the mean level spacing[24]. It was found that the oneelectron eigenstates become extended for transfer terms decaying slower than $1 / r^{2}$. Finally, the Anderson transition in a $1 D$ chain with random power-law decaying hopping terms and non-random on-site energies was numerically characterized in ref. [25].

Another class of models presenting an Anderson localization corresponds to models for one-electron moving in diluted lattices. The states present the so called quantum percolation transition separating regimes of extended and localized states. The quantum percolation threshold is usually different from the classical percolation point once disorder in more effective in localizing wave-like than particle-like excitations. In this work, we investigate the nature of one-electron eigenstates in power-law diluted chains for which the probability of occurrence of a bond between sites separated by a distance $r$ decays as $p(r)=1 / r^{1+\sigma}$. Although this chain is fully connected through the first-neighbors couplings and, therefore, allows for classical percolation, we will show that the disorder introduced by the dilution of long-range hoppings may localize the one-electron eigenstates. Using an exact diagonalization 
scheme, we will provide a detailed analysis of the nature of the one-electron eigenstates and the wave-packet dynamics. Using a finite-size scaling analysis, we will also locate the critical power-law exponent below which extended states emerge in this model.

\section{MODEL AND FORMALISM}

\section{A. Model Hamiltonian}

We consider a single electron in a 1D chain with open boundaries, described by the Anderson Hamiltonian

$$
H=\sum_{n=1}^{N} \varepsilon_{n}|n\rangle\left\langle n\left|+\sum_{n \neq m}^{N} h(|n-m|)\right| n\right\rangle\langle m|,
$$

where $|n\rangle$ represents the state with the electron localized at site $n$. In the present random bond Anderson model, the on-site potentials $\varepsilon_{n}$ are site independent and in Eq. 1 were taken to be $\varepsilon_{n}=0$ without any loss of generality. Long-range disorder is introduced by assuming the hopping amplitudes $h(n-m)$ to be distributed following a power-law decaying distribution. The probability of occurrence of a bond between sites $n$ and $m$ decays as

$$
p(r=|n-m|)=1 / r^{1+\sigma} .
$$

where $h(|n-m|)=1$ with probability $p(r)$ and $h(|n-m|)=0$ with probability $1-p(r)$. For $\sigma>1$ this model is expected to have features similar to those presented by models with random short-range couplings. In this work, we will be particularly interested in the regime of $0<\sigma<1$.

\section{B. Eigenfunctions and Participation number}

In the following investigation, we will analyze the quantum percolation transition in the above introduced model. The main quantities we used for such purpose were obtained by the direct diagonalization of the Hamiltonian on finite chains which provided all eigenstates and energy eigenvalues for each disorder realization. In studying the nature of the oneelectron eigenstates, the participation moments play a central role. For a particular disorder configuration $v$ and eigenstate, the participation number is defined as the inverse of the second moment of the probability density

$$
P^{j, v}=\frac{1}{\sum_{n=1}^{N}\left|f_{n}^{j, v}\right|^{4}}
$$

where $f_{n}^{j, v}$ is the amplitude at site $n$ of the $j$-th eigenstate from the $v$-th disorder realization. In our numerical computation, we performed a numerically exact diagonalization on chains with sizes ranging from $N=200$ up to $N=1600$ sites. We averaged $P^{j, v}$ using all eigestates computed from distinct disorder realizations:

$$
\langle\xi\rangle=\frac{1}{M N} \sum_{v=1}^{M} \sum_{j=1}^{N} P^{j, v} .
$$
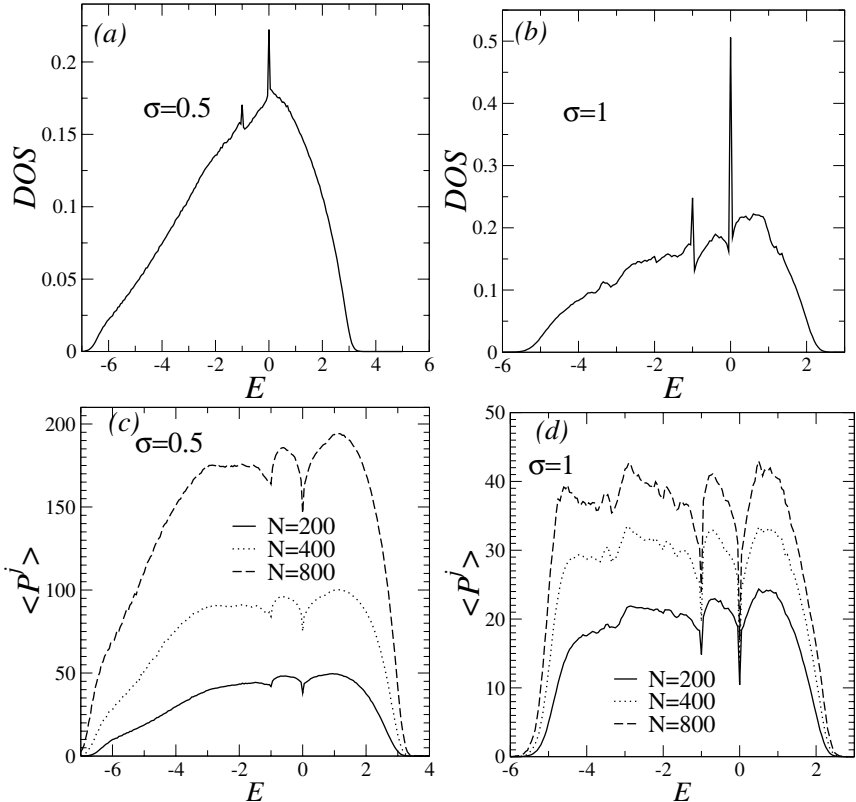

FIG. 1: The normalized density of states DOS versus energy $E$ for $N=200$ and (a) $\sigma=0.5$, (b) $\sigma=1$. Results for DOS were averaged using more than 30000 disorder realizations. The participation number $\left\langle P^{j}\right\rangle$ (averaged over several realizations of disorder) versus energy $E$ for several system sizes $(N=200$ up to 800), (c) $\sigma=0.5$ and (d) $\sigma=1$. For $\sigma=0.5$ the participation number is proportional do $N$ indicating the presence of extended states. The participation number converges to finite values for $\sigma=1$. This result suggest a localized nature of all eigentates.

We use $N M=64 \times 10^{3}$ states for each chain size. In addition, we computed the normalized density of states (DOS) defined by $\operatorname{DOS}(E)=(1 / M N) \sum_{j, v} \delta\left(E-E^{j, v}\right)$ and the fluctuation of the participation number defined by

$$
\Delta \xi=\sqrt{\left\langle\xi^{2}\right\rangle-\langle\xi\rangle^{2}}
$$

The relative fluctuation of the participation number is given by

$$
\eta=\Delta \xi /\langle\xi\rangle
$$

Within the framework of random and non-random long-range hopping models, it was demonstrated rigorously that the distribution function of the participation function is scale invariant at the Anderson transition [26]. Such scale invariance has been used to monitor the critical point of long-range hopping models [27] and shall also hold for general models exhibiting a localization-delocalization transition.

\section{Wave-packet Dynamics}

In order to obtain the time-evolution of an initially localized wave-packet $(|\Phi(t=0)\rangle)$, we expanded the wave-function in the Wannier representation 

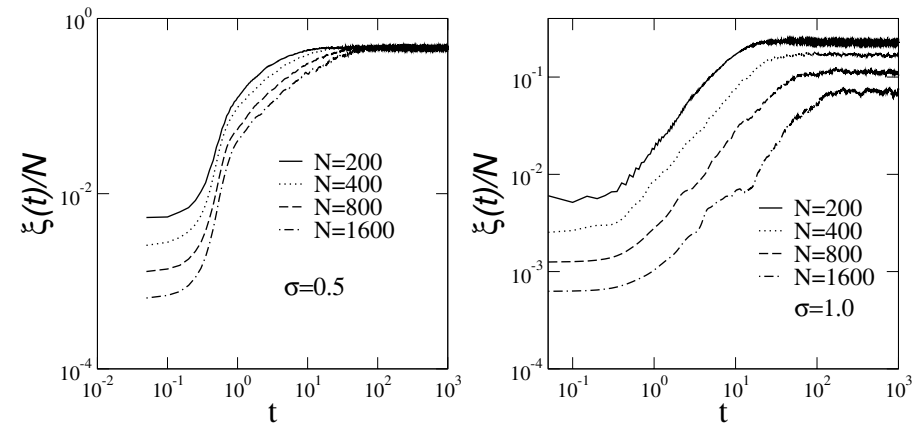

FIG. 2: Scaled participation number $\xi / N$ versus time $t$ for several system sizes ( $N=200$ up to 1600$),(a) \sigma=0.5$ and (b) $\sigma=1$. For $\sigma=0.5$ the asymptotic participation number is proportional to $N$ indicating the presence of extended states among the wave-packet components. For $\sigma=1$, the Scaled participation number $\xi / N$ tends to zero as the system size is increased. This result suggests that all eigenstates are localized.

$$
|\Phi(t)\rangle=\sum_{n} f_{n}(t)|n\rangle .
$$

The main task is to solve the time-dependent Schrödinger equation for the wave-function components $f_{n}(t)(\hbar=1)$

$$
i \frac{d f_{n}(t)}{d t}=\sum_{n \neq m}^{N} h(|n-m|) f_{n}(t) .
$$

Using the numerical formalism proposed in Ref. [28], the general solution of this problem can be written as:

$$
|\Phi(t)\rangle=\mathbf{U}^{\dagger} \exp (-i \mathbf{D} t) \mathbf{U}|\Phi(t=0)\rangle,
$$

where $\mathbf{D}$ is the diagonal form of the Hamiltonian and $\mathbf{U}$ is a unitary matrix. In what follows, we consider the electron initially localized in a single site, i.e. $|\Phi(t=0)\rangle=\left|n_{0}\right\rangle$.

Besides following the time evolution of the initially localized wave-packet, we also recorded the asymptotic scaled return probability (return probability times the chain size) defined as:

$$
R=\frac{N}{t_{\infty}} \int_{0}^{t_{\infty}}\left|f_{n_{0}}(t)\right|^{2} d t
$$

In general $R$ is roughly a constant for extended states and scales proportional to $N$ for localized states. We further calculated the time dependent participation number

$$
\xi(t)=\left\langle\frac{1}{\sum_{n}^{N}\left|f_{n}(t)\right|^{4}}\right\rangle,
$$

averaged over distinct realizations. The participation number $(\xi(t))$ has been commonly used as a measurement of the typical number of sites over which the wave-packet is spread.

\section{RESULTS}

In Fig.1, we show results for the normalized density of states DOS and the participation number. In Fig.1a, we plot

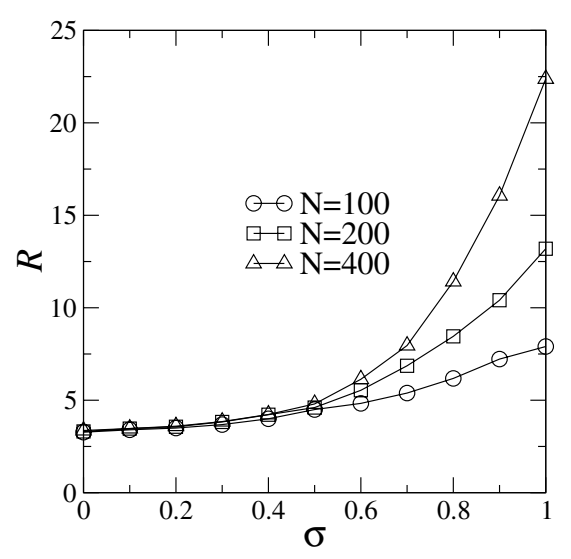

FIG. 3: The asymptotic scaled return probability $R$ versus $\sigma$ for several system sizes ( $N=100$ up to 400$)$. The scaled return probability $R$ is roughly a constant for small $\sigma$ and increase with $N$ for $\sigma$ around unit. This result reinforces the existence of an Anderson transition for $\sigma<1$

the normalized density of states DOS versus energy $E$ for $N=200$ and $\sigma=0.5$. In Fig. $1 \mathrm{~b}$, similar data are shown for $\sigma=1$. Results for the DOS were averaged using more than 30000 disorder realizations. In both cases, two delta-like singularities are observed at $E=0$ and $E=-1$. These singularities are associated with resonant localized modes which are located at pair of sites with the same bonds to the remaining chain. It is important to notice that for $\alpha=0.5$ the DOS presents much smaller fluctuations than that for $\alpha=1$. This feature signals that the typical localization length becomes larger as the couplings are made more long-ranged.

The average participation number $\left\langle P^{j}\right\rangle$ versus energy $E$ for several system sizes $(N=200$ up to 800$)$ is showed in Fig.1(c) for the particular case of $\sigma=0.5$. Fig.1(d) show our results for $\sigma=1$. To compute $\left\langle P^{j}\right\rangle$, we averaged the participation number of all eigenstates $j$ within a small window around $E$ considering several disorder realizations. For $\sigma=0.5$ the participation number is proportional to $N$ indicating the presence of extended states. The average participation number does not scale proportional to the system size for $\sigma=1$. This result suggests an asymptotic localized nature of all eigentates. The dips at $E=0$ and $E=-1$ are due to the strongly localized nature of the resonant states at these energies.

In Fig.2, we show our results for the scaled participation number $\xi / N$ versus time $t$ for several system sizes $(N=200$ up to 1600 ), (a) $\sigma=0.5$ and (b) $\sigma=1$. For $\sigma=0.5$ the asymptotic participation number is proportional to $N$ indicating that the wave-packet has components which are extended states. For $\sigma=1$, the scaled participation number $\xi / N$ tends to zero as the system size is increased. This result suggests that all eigenstates have a localized nature. It is important to stress that the asymptotic regime for $\sigma=0.5$ is reached after a very short evolution time. Due to the presence of long-range hoppings, the initially localized wave-packet spreads exponentially before the saturation.

The above results indicates that a quantum percolation transition is taking place in such power-law diluted chain model. 


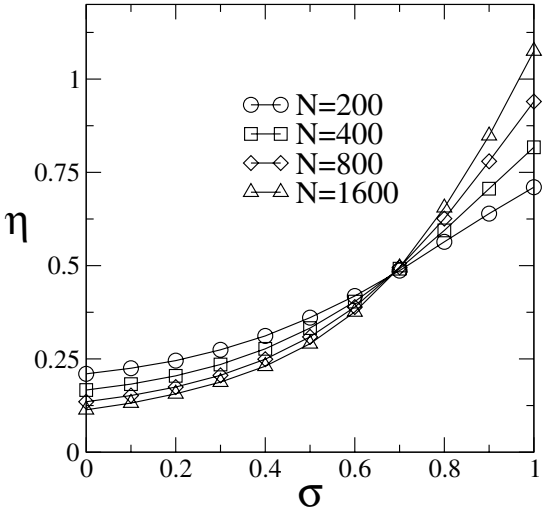

FIG. 4: The relative fluctuation of the participation function $\eta=$ $\Delta \xi /\langle\xi>$ versus $\sigma$ and $N=200,400,800,1600$ sites. The scale invariant point signals the transition between extend and exponentially localized states.

The main parameter governing this transition is the power-law decay exponent $\sigma$. In order to locate the critical decay exponent, we computed some typical quantities over the full range of $0<\sigma<1$. In Fig.3 we show the asymptotic scaled return probability $R$ versus $\sigma$ for distinct system sizes $(N=100$ up to 400$)$. The scaled return probability $R$ is roughly a constant for small $\sigma$ and increase with $N$ for large $\sigma$. In agreement with Fig. 2, this result also indicates the existence of extended states for $\sigma=0.5$. However, due to the strong finite size effects, such quantity does not allow for an accurate estimate of the critical point. To this end, the relative fluctuation of the participation number plotted as a function of $\sigma$ using data computed from distinct chain sizes represents one of the most efficient and computationally suited tools. Extended states display very weak participation number fluctuations and, as such, the relative fluctuation decreases with the system size. On the other hand, localized states have size independent fluctuations with the relative value saturating at a finite value as the system size is increased. At the critical point, the participation number distribution is scale invariant. In Fig.4, we collect results of the relative fluctuation of the participation function $\eta=\Delta \xi /<\xi>$ versus $\sigma$ with $N=200,400,800,1600$ sites. The scale invariant point around $\sigma=0.68(2)$ signals the transition between extended and exponentially localized states.

\section{SUMMARY AND CONCLUSIONS}

In summary, we investigated the nature of one-electron eigenstates in power-law diluted chains for which the prob- ability of occurrence of a bond between sites separated by a distance $r$ decays a $p(r)=1 / r^{1+\sigma}$. This model is a prototype to study the quantum percolation transition in lowdimensional systems with long-range couplings. Using an exact diagonalization scheme on finite chains, we computed the DOS and participation number of all energy eigenstates which were used to follow the time-evolution of an initially localized wave-packet. We found that the relative fluctuation of the participation number provides a precise estimate of the critical decay exponent $\sigma_{c}=0.68$ (2) separating the regimes of localized and extended states. For decay exponents larger than this critical value, all energy eigenstates are localized, a behavior typical of disordered systems with short-range couplings. It is important to stress that in this regime the wave-packet remains localized over a finite region of the system even though the chain is fully connected. This is a typical signature of systems presenting classical percolation but no quantum percolation. For $\sigma$ below the critical value, the long-range couplings are enough to stabilize extended states. An initially wave-packet develops a fast exponential spread over the chain. As compared with other classes of models with long-range couplings, such as the random band matrix model and models with longrange couplings and pure diagonal disorder, the present model is the one on which the phase of extended states is sustained for the widest range of decay exponents. Therefore, there is a range of decay exponents for which the present quantum percolation model exhibits a localization-delocalization transition that is not shared by these other models. A $\sigma$-dependent set of new critical exponents shall govern this unique transition. Within this scenario, the quantum percolation transition here reported is expected to belong to a universality class distinct from the two classes governing the Anderson transition in models with long-range couplings with pure diagonal as well as off-diagonal disorder. We expect the present results can stimulate further studies along this direction.

\section{ACKNOWLEDGEMENTS}

This work was partially supported by the Brazilian research agencies CNPq, Rede Nanobioestruturas, CAPES and FINEP and by the Alagoas State research agency FAPEAL.
[1] P.W. Anderson Phys. Rev. 109, (1958) 1492.

[2] E. Abrahams, P.W. Anderson, D.C. Licciardello, and T.V. Ramakrishnan, Phys. Rev. Lett. 42, 673 (1979). For a review see, e.g., I.M. Lifshitz, S.A. Gredeskul, and L.A. Pastur, Introduc- tion to the Theory of Disordered Systems (Wiley, New York, 1988).

[3] A.D. Mirlin, Y.V. Fyodorov, F.-M. Dittes, J. Quezada, and T.H. Seligman, Phys. Rev. E 54, 3221 (1996). 
[4] A.D. Mirlin and F. Evers, Phys. Rev. B 62, 7920 (2000); F. Evers and A.D. Mirlin, Phys. Rev. Lett. 84, 3690 (2000).

[5] J.C. Flores, J. Phys.: Condens. Matter 1, 8471 (1989).

[6] D.H. Dunlap, H.L. Wu, and P.W. Phillips, Phys. Rev. Lett. 65, 88 (1990); H.-L. Wu and P. Phillips, Phys. Rev. Lett. 66, 1366 (1991); P.W. Phillips and H.-L. Wu, Science 252, 1805 (1991);

[7] C.A.A. da Silva, P.E. de Brito, and H. N. Nazareno, Phys. Rev. B 52, 7775 (1995).

[8] V. Bellani, E. Diez, R. Hey, L. Toni, L. Tarricone, G.B. Parravicini, F. Domínguez-Adame, and R. Gómez-Alcalá, Phys. Rev. Lett. 82, 2159 (1999).

[9] F.A.B.F. de Moura and M.L. Lyra, Phys. Rev. Lett. 81, 3735 (1998); F.A.B.F. de Moura and M.L. Lyra, Physica A 266, 465 (1999).

[10] F.M. Izrailev and A.A. Krokhin, Phys. Rev. Lett. 82, 4062 (1999); F.M. Izrailev, A.A. Krokhin, and S.E. Ulloa, Phys. Rev. B 63, 041102(R) (2001).

[11] F.A.B.F. de Moura, M.D. Coutinho-Filho, E.P. Raposo, and M.L. Lyra, Europhys. Lett. 66, 585 (2004).

[12] P.E. de Brito, C.A.A. da Silva, and H.N. Nazareno, Phys. Rev. B 51, 6096 (1995).

[13] H.N. Nazareno, P.E. de Brito, and C.A.A. da Silva, Phys. Rev. B 51, 864 (1995).

[14] C.S. Ryu, G.Y. Oh, and M.H. Lee, Phys. Rev. B 48, 132 (1993).

[15] H.N. Nazareno and P.E. de Brito, Phys. Rev. B 60, 4629 (1999).

[16] P.E. de Brito, E.S. Rodrigues, and H.N. Nazareno, Phys. Rev. B 69, 214204 (2004)
[17] A. Rodríguez, V.A. Malyshev, and F. Domínguez-Adame, J. Phys. A: Math. Gen. 33, L161 (2000).

[18] A. Rodríguez, V.A. Malyshev, G. Sierra, M.A. Martín-Delgado, J. Rodríguez-Laguna, and F. Domínguez-Adame, Phys. Rev. Lett. 90, 27404 (2003).

[19] A.V. Malyshev, V.A. Malyshev, and F. Domínguez-Adame, Phys. Rev. B 70, 172202 (2004).

[20] R.P.A. Lima, M.L. Lyra, and J.C. Cressoni, Physica A 295, 154 (2001).

[21] F.M. Izrailev, T. Kottos, A. Politi, S. Ruffo, and G.P. Tsironis, Europhys. Lett. 34, 441 (1996).

[22] F.M. Izrailev, T. Kottos, A. Politi, and G.P. Tsironis, Phys. Rev. E 55, 4951 (1997).

[23] A. Politi, S. Ruffo, and L. Tessieri, Eur. Phys. J. B 14, 673 (2000).

[24] H. Potempa and L. Schweitzer, Phys. Rev. B 65, 201105(R) (2001).

[25] R.P.A. Lima, H.R. da Cruz, J.C. Cressoni, and M.L. Lyra, Phys. Rev. B 69, 165117 (2004).

[26] F. Evers and A. D. Mirlin, Phys. Rev. Lett. 84, 3690 (2000); ibid Phys. Rev. B 62, 7920 (2000).

[27] A. V. Malyshev, V. A. Malyshev, and F. Domínguez-Adame, Phys. Rev. B 70, 172202 (2004).

[28] H.N. Nazareno, P.E. de Brito, and E.S. Rodriguez, Phys. Rev. B 68, 054204 (2003). 